\title{
A Digital Workflow for an Implant Retained over Denture: A New Approach
}

\author{
Luca Ortensi ${ }^{* 1}$, Riccardo Stefani ${ }^{2}$, Luca Lavorgna ${ }^{3}$, Ilaria Caviggioli ${ }^{4}$ and Tommaso Vitali $^{5}$ \\ ${ }^{1}$ Department of Prosthodontics, University of Catania, Italy \\ ${ }^{2}$ Private practice, San Donà di Piave, Italy \\ ${ }^{3}$ Private practice, Telese, Italy \\ ${ }^{4}$ Private practice, Galliate, Italy \\ ${ }^{5}$ Private practice, Castiglione del Lago, Italy
}

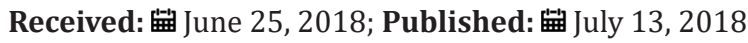

*Corresponding author: Luca Ortensi, Via De Gombruti, 540123, Bologna, Italy

\section{Abstract}

The development of digital technologies in dentistry has changed the therapeutic approach in edentulous patients both in the preliminary stages of clinical case studies and when supporting the actual fabrication phases. The goals of digital fabrication are to reduce the number of patient appointments and improve the predictability of treatment outcome. A thorough description of a complex clinical case analyzes how these new digital technologies are used in every step of the prosthetic therapy to perform the reconstruction of a bar retained overdenture.

\section{Introduction}

For some years, modern prosthetic dentistry has been making use of digital technologies to support both the diagnostic and therapeutic stages of patient rehabilitation [1-4]. The traditional removable and implant-supported denture has also benefited from these innovations and has been further digitally developed both in the virtual planning of clinical cases and as support during the actual fabrication phase $[5,6]$. The purpose of digital fabrication of complete dentures and overdentures is to reduce the number of patient appointments and improve the predictability of treatment outcome [7]. Nonetheless, no standardized procedure has been determined for the digital fabrication of removable prostheses starting from the communication with patients to the construction of prostheses in the dental laboratory. Current analogous work steps and digital fabrication approaches have yet to be integrated and linked to one complete digital workflow. For this reason a new area of application for CAD technology is presented for prosthetic treatment, particularly for bar retained overdentures through a new specific software called Digital Smile SystemR(DSS, Digital Smile System DSS).

DSS is a software composed of tools and a data library of natural and artificial teeth used in interdisciplinary dentistry to improve diagnostic vision, develop communication/information, and enhance predictability throughout the course of treatment (restorative, surgical, and prosthetic) [8,9]. This system employs two photographs of the patient's face that can be processed to create a clinical, functional, and esthetic design of the smile. DSS is used not only as a means for dentist/patient dialogue, but also as a work tool for transferring to the dental technician all the information needed to create the prosthetic artefact, limiting to a minimum both the clinical trials and the analogue laboratory stages.The aim of this clinical report is to describe the application of these digital technology advancements in overdenture implant rehabilitation through the description of a complex clinical case.

\section{Clinical Report}

A 68-year-old female patient came to the dental office complaining of reduced chewing ability and loss of retention of her upper removable complete denture present in the oral cavity. She furthermore asked to improve the esthetics of her smile and face, lamenting that she was dissatisfied with the color and visibility of her teeth which, even when facial mimicry was more accentuated, were scarcely noticeable and featured unnatural slanted planes. The medical history showed no pathology incompatible with dental therapy and an overall good state of health: the patient was classified as ASA1.The esthetic analysis of the facial appearance showed a reduction of the vertical dimension of occlusion with an increase in perilabial wrinkles and the upper teeth were not visible in the rest position. Furthermore, the musculature of the cheeks was unsupported by the old prosthesis, and the entire face had visibly lost tone (Figure 1). The patient exhibited an unpleasant, slanted smile with a disharmonious aspect.The clinical intraoral analysis showed that the upper denture was unsatisfactory because of an inadequate extension of the prosthetic bodies, incorrect occlusal contacts, and an inadequate intermaxillary relationship. In 
the lower arch, a recently produced bar retained overdenture was present.

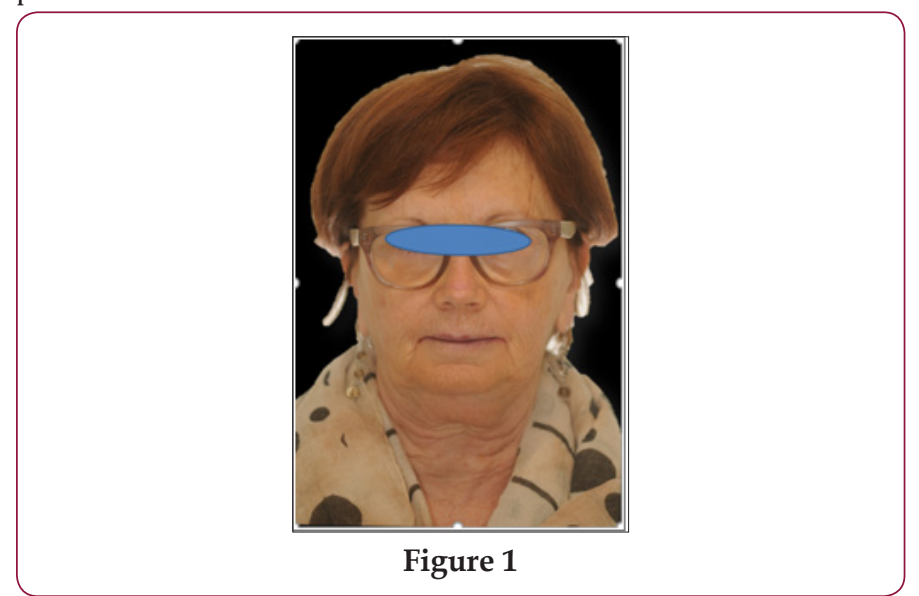

During the visit, the patient underwent a lateral radiographic exam. The radiographic examination made it possible to study the hard and soft tissues of the patient's face, particularly the relationship between the maxillary, the spatial position of the upper central incisor, and the philtrum, providing prognostic information for treatment planning purposes [6-8]. Facial and intraoral photographs were taken as an essential aid for completing the treatment plan. In general, facial photos enable further diagnostic assessments regarding the overall esthetics of the face and the physiognomic traits, which are then addressed in the prosthetic therapy[9].At the end of the first visit, different treatment options with respective associated risks and benefits were presented and discussed with the patient. The patient accepted the definitive treatment plan of an overdenture maxillary dental prosthesis implant, supported with four dental implants.The first clinical stage of the new prosthetic therapy entails making the preliminary impressions. This is in no way a marginal step in the process as it is essential to fully record the anatomy of the endoral structures of the edentulous upper jaw.

A digital impression of the edentulous upper arch was not used because this procedure is not yet perfectly coded for edentulous patients $[10,11]$. Only digital impressions for the opposite arch were used. In the upper archa high-precision alginate (Hydrogum5, Zhermack Spa) was used in two phases: the first impression was made using a mixed, high-consistency alginate, which was then dried and modified using a sharp instrument to eliminate the undercut. It was then rebased with the same material but in a more fluid form in order to read all the details of the anatomical tissues. From the preliminary impression an extra-hard plaster model was obtained. The master plaster model was digitally acquired with a scanner (Sinergia Scan, Nobil Metal Spa),and the files obtained used afterwards. In the case at hand, an occlusal rim was constructed directly onto the initial model [12] and then functionalized in the oral cavity. It was important to mark a few landmarks in the occlusal rim: the median line, the canine line, and the smile line. These reference lines are used by the DSS software during the imagining aligning phase.

At this point two photos were taken of the patient's face with the occlusal rim inserted in the oral cavity, according to a coded technique for the DSS software [13]. It is important to take photos of the face keeping the patient in a position that is stable and repeatable over time, trying not to change the enlargement ratio between shots. For this purpose, the patient was invited to sit comfortably keeping her back erect while the operator used a camera set on a tripod to stabilize its position with respect to the patient being photographed. The subject had to be positioned so that her Frankfurt Plane (the line that joins the Porion and the Orbital Point) was parallel to the horizon.Once the spatial position of the cranium has been identified, it must stay unchanged with respect to the camera and tripod. The patient may wear dedicated glasses used to calibrate the digital pre-rendering software (DSS). The glasses represent a true measuring system that differentiates this software from other similar systems [14].

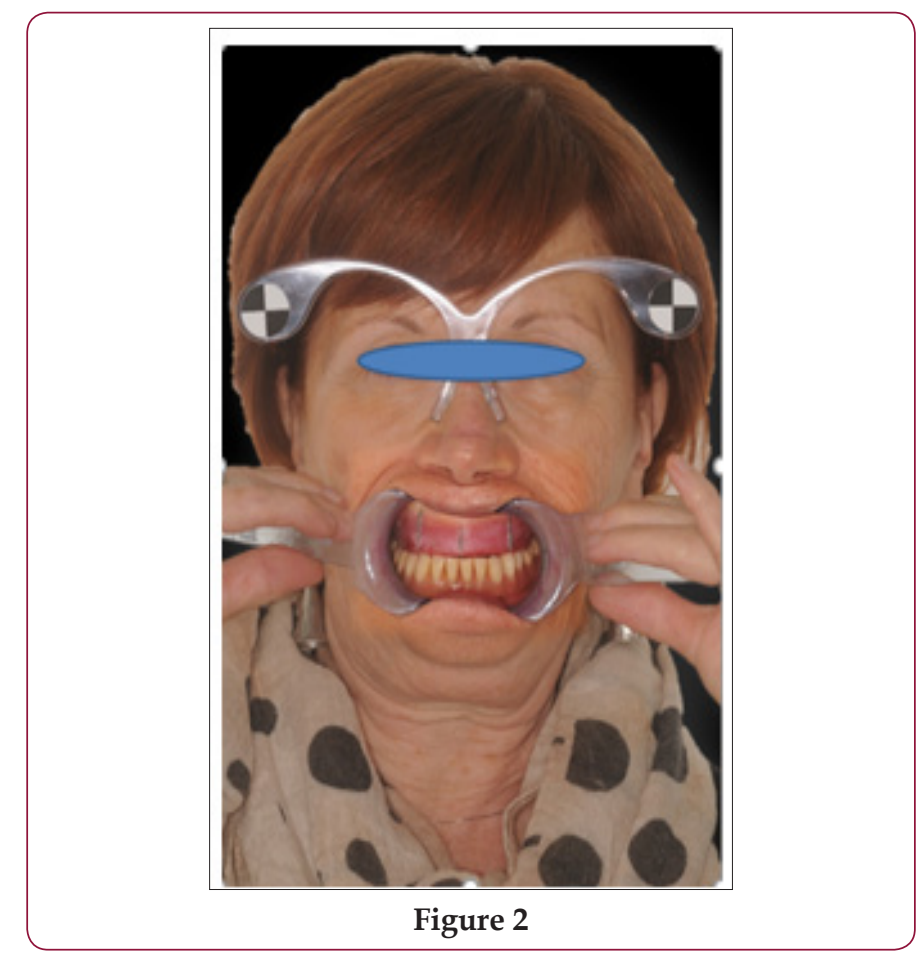

The first facial photo was taken asking the patient to smile and show as many teeth as possible. This shot depicted how the incisal plane was developing with respect to the lower lip and the width of the lateral corridors. The second facial photograph was taken with cheek retractors (Figure 2). The photo made it possible to correctly assess the parallelism between the bipupillary plane and the occlusal plane, as well as the consistency between the median and interincisive line. This made it possible to import the photos (JPEG file format) into the DSS and proceed with the esthetic pre-rendering of the future prosthetic therapy. Digital pre-rendering with the DSS program consisted of creating an digital teeth arrangement with artificial teeth contained in the software library. The teeth were chosen according to esthetic and functional parameters [15] and could be replaced with others of different shapes or color, if necessary. The anterior and posterior teeth were positioned using the occlusal rim, suitably adapted in the oral cavity beforehand, as a guide. In this way the patient could see the possible esthetic end result and participate in the therapeutic project together with the entire dental team (Figures $3 \& 4$ ). 


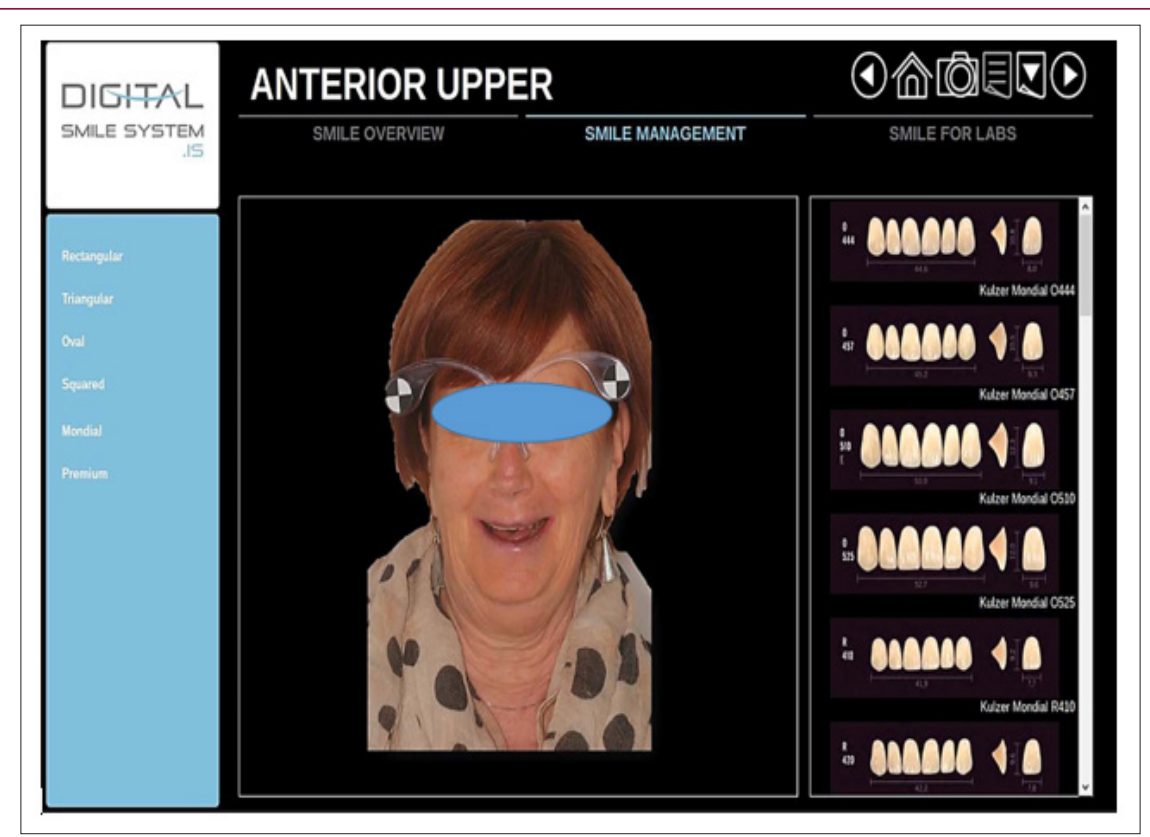

Figure 3

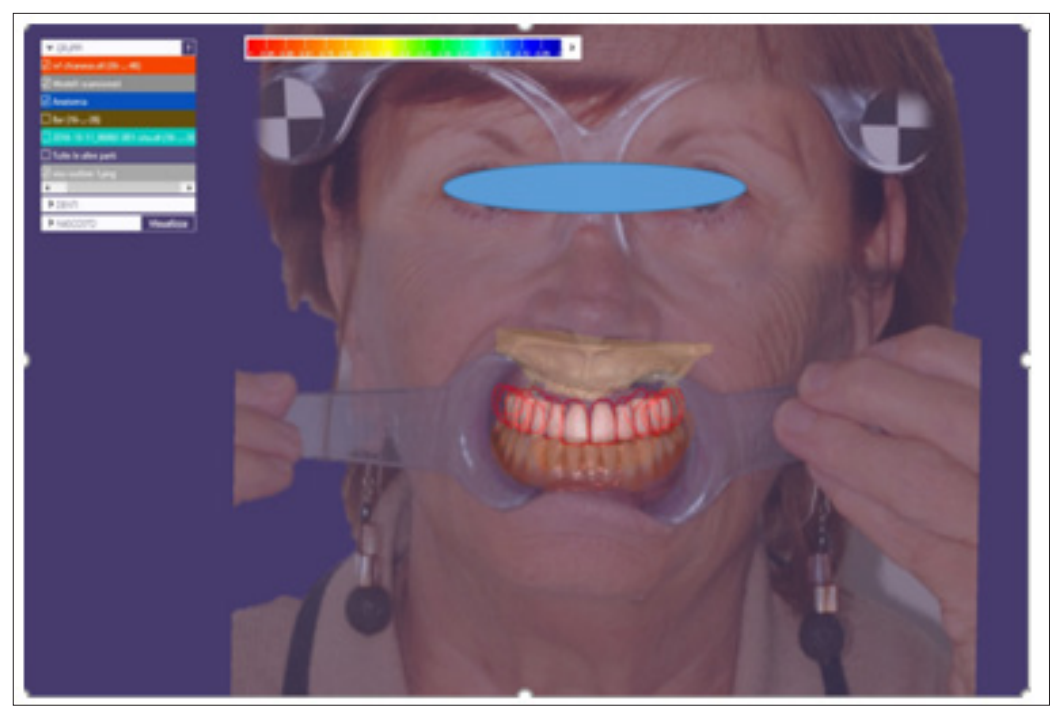

Figure 4

Once the virtual teeth arrangement were obtained - and approved by the patient - the file containing the patient's information, the photographic alignments, the libraries chosen, and the work process was transferred to the dental technician's laboratory where the file was imported into a 3D software (Exocad ${ }^{\circledR}$ software, Exocad $\mathrm{GmbH}$ ). The information file exported from the DSS consisted of a PDF format and single photographs of the patient's face with a customized two dimensional (2D) virtual smile design.Subsequently the occlusal rim was scanned (Sinergia Scan, Nobil Metal Spa) and imported into Exocad. The files from DSS were then superimposed on scanned images of the rim and upper stone model [16]. The dental technician used the outline of the virtual smile obtained to place a tooth from the library or create customized teeth with tools from Freeform (Exocad $\AA$ ) software, Exocad $\mathrm{GmbH}$ ) to convert the virtual 2D teeth arrangement into a 3D teeth arrangement [17].
The 3D phase was then completed by improving the occlusal relation between the virtual teeth and the opposite arch using the occlusal rim as a guide. At the end of this work stage, a file from Exocad was converted into a specific file At the end of this work stage, a file from Exocad was converted into a specific file for a Cad-Cam technology (Roland DWX50) and a prototype of the digital work was produced with a dedicated wax (Wax Disc grey, Dentsplay Sirona) [18]. The prototype was tried in the mouth checking the intraoral adaptation, the centric relation, and the esthetics of the smile and face (Figure 5). The clinician could make changes if necessary. Subsequently four implants (Thommen Medical 4.0/6,5mm) were placed in the upper jaw using prototypes like a surgical guide with a one stage surgery. The healing abutments were positioned directly on the implant heads and the old denture was relined with a temporary soft material (Coe Soft (CS) GC America Inc.).After four weeks [19], the final digital impression (Trios 3Shape) of the upper 
arch was made of the implant scan bodies previously screwed into the fixture, with a set torque. In addition, the final volume of the clinicized prototype were also reacquired so as to digitally superimpose the images onto each other. The superimposition of various digital files has proven to be a reliable procedure in digital work flows (Figure 6)[20].
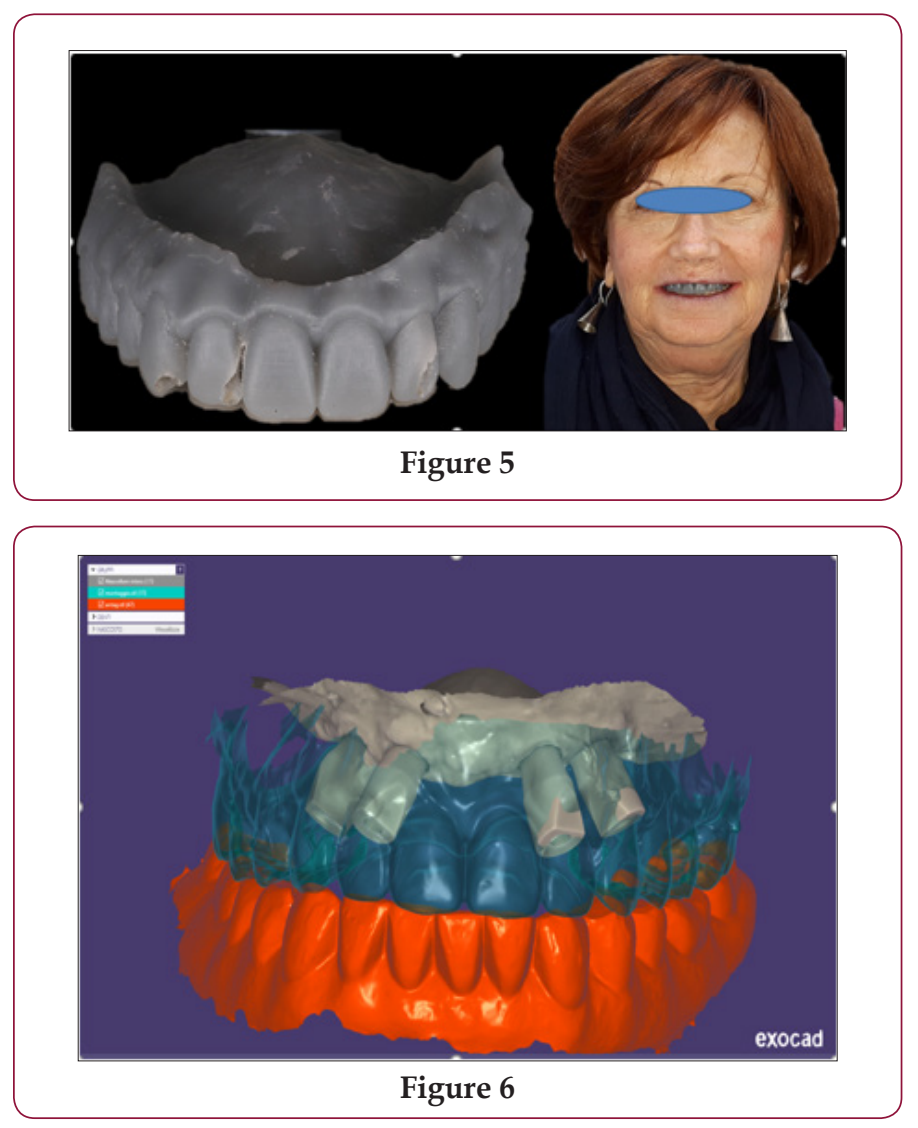

Using a dedicated tool of the 3D software (Exocad® software, Exocad $\mathrm{GmbH}$ ), the project was executed to create the upper retentive bar, using the fixture scan transparencies to assess the available spaces and the position of the teeth with respect to their analogues, and identify the type of prosthetic structure and anchorage. In the designed bar there were four anchorage areas: two in the front and two located distally.The structure project was sent to the milling center (New Ancorvis): indications were given regarding the retentive system anchorage areas, the use of dedicated threading, the type of metal, and the execution technique (CAD/ CAM technology).After being checked in the dental technician's lab, the handwork was sent to the dentist to clinically test the structure, in order to verify its precision and passivity.The dentist took a pick up impression of the bar so that it was possible to make a master model stone to finalize the prosthesis. Then the technician proceeded with an initial polishing of the artefact and the screwing in of the retentive systems (Micro screwed-in OT cap attachments, Rhein 83 ). The process later continued with further polishing and shining of the bar and the digital acquisition using a scanner.

The dental technician digitally designed the counter-bar, always checking the available spaces, using final volume superimposition and inserting retentive pins in the project for the mechanical tightness of the teeth. Fabrication was carried out using laser- melting technology that made it possible to obtain an accurate handmade to which the acrylic resin adhered tightly thanks to the presence of a retentive surface [21]. It was then time for the complete application of the teeth onto the counter-bar, using the clinicized prototype as a fitting plane. The joining of the artificial teeth and the metal structure was carried out using a small amount of wax (Figure 7). The prosthesis was finalized using a resin injection muffle system (VertysystemR, a-gree srl) that transformed the wax into resin with a codified protocol $[22,23]$. The overdenture and the finished and perfectly polished retention structure were sent to the dental office (Figure 8). The bar was then inserted into the oral cavity by the clinician and tightened to the implant fixtures with a preset torque.


The dentist had to check that there were no areas where the soft tissues were compressed and that there was room for the use of dental devices: interdental brushes and flosses. Notice the 
parallelism of the retentive components which is synonymous with hold and maintainability over time (Figure 9). Once the prosthetic therapy was completed, the patient's face had improved greatly from an esthetic viewpoint. The soft tissues of the face appeared firm and toned. A reduction in the naso-labial folds and perilabial wrinkles was noticed, both frontally and laterally. The vertical dimension, which had slightly been increased, appeared adequate and well tolerated esthetically. During phonation and smiling dynamics the patient displayed natural looking teeth that were perfectly integrated with the face (Figure 10).

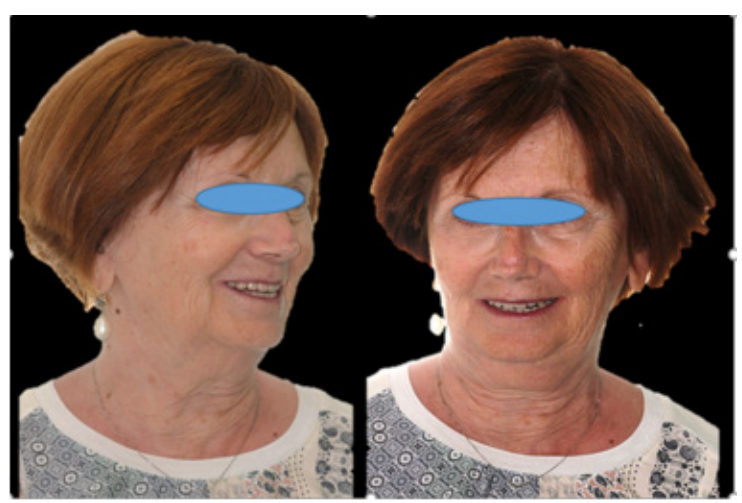

Figure 10

\section{Discussion}

In prosthetic therapy, for both fixed and removable prostheses, communication with the patient is a vital part of the treatment. Effective digital previsualization is the ideal way to explain esthetic changes to a patient and receive their approval. Until now, many digital previsualization methods have been used in dentistry solely with this scope.In this article the digital smile approach was introduced in a complex digital workflow. DSS not only allowed the patient to see her future aesthetics, but it also permitted to produce a prototype for the functional check of the digital project carried out. The fact that the patient can see the possible future aesthetic results through digital rendering, asking for changes if desired, reduces overall clinical practice time. Additionally, the construction of a prototype, based on the virtual assembly, minimizes the number of errors in the fabrication of the final product.

Some phases of the workflow require a learning curve on behalf of the clinical operator and technician. For example, the photos taken by the clinician for the DSS, must be executed in the exact manner in which previously described to facilitate the superimposing of the photo of the patient's face, with the scan of the model and the occlusal rim. Another important stage is that in which the teeth from the databank of the 3D software are matched with the outlines obtained by digital previsualization with DSS. In this case, if the matching is not precise, the prototype will not correspond perfectly with the one approved by the patient.

\section{Conclusion}

The use of digital technologies is now vastly widespread in the field of dentistry and, in particular, in prosthetic therapy. In removable, traditional, and implant support prosthetic therapy, digital technology can play an essential role. The clinical case described was almost entirely resolved with an innovative digital workflow, both from a clinical and technical viewpoint. The human component is still fundamental and not all stages can be carried out digitally. However, it is expected that a technical evolution will rapidly lead to more and more digitalized therapies with an increase in the end quality of the therapy and less conditioned by the skills of the individual operator.

\section{Acknowledgment}

The authors thank Mr Gianni Ortensi, CDT, Mr. Marco Ortensi, CDT, Mr. Carlo Borromeo, CDT, Mr Cesare Chiarini, CDT, and Mr. Fabrizio Molinelli, CDT, who fabricated the bar retained overdenture.

\section{References}

1. Coachman C, Calamita M (2014) Virtual Esthetic Smile Design. J Cosmet Dent 29(4): 102-116.

2. Caviggioli I, Molinelli F, Ortensi L, Stefani R (2011) La prima visita in odontoiatria protesica: aspetti innovativi. Il Dentista Modernogiugno.

3. Zimmermann M, Mehl A (2015) Virtual smile design systems: a current review. Int J Computer Dent 18(4): 303-317.

4. Coachman C, Calamita M (2012) A Tool for Treatment Planning and Communication in Esthetic Dentistry QDT.

5. Goodacre CJ, Garbacea A, Naylor WP, Daher T, Marchack CB, Lowry J (2012) CAD/CAM fabricated complete dentures: concepts and clinical methods of obtaining required morphological data. J Prosthet Dent 107(1): 34-46.

6. Infante L, Yilmaz B, Mc Glumphy E, Finger I (2014) Fabricating complete dentures with CAD/CAM technology. J Prosthet Dent 111(5): 351-355.

7. Kattadiyil MT, Jekki R, Goodacre CJ, Baba NZ (2015) Comparison of treatment outcomes in digital and conventional complete removable dental prosthesis fabrications in a predoctoral setting. J Prosthet Dent 114(6): 818-825

8. Rossi M, Molinelli F, Caviggioli I (2015) Latest trends in prosthetics-Total maxillary rehabilitation with a Toronto Bridge using digital technologies. CAD/CAM International magazine of digital dentistry 1: 28-31.

9. McLaren EA, Terry D (2001) Photography in dentistry. J Calif Dental Ass 29(10): 735-742.

10. Kim JE, Amelya A, Shin Y, Shim JS (2017) Accuracy of intraoral digital impressions using an artificial landmark. J Prosthet Dent 117(6): 755761.

11. Patzelt SB, Vonau S, Stampf S, Att W (2013) Assessing the feasibility and accuracy of digitizing edentulous jaws. The Journal of the American Dental Association 144(8): 914-920.

12. Schreinemakers J (1979) Die Logik in der Totalprothetik. Quintessenzp. 360.

13. Stefani R, Caviggioli I, Molinelli F, Ortensi L (2012) L'impiego delle tecnologie digitali nella diagnosi protesica e nella realizzazione della protesi. Il Dentista Moderno Ottobre.

14. Jokstad A (2017) Computer-assisted technologies used in oral rehabilitation and the clinical documentation of alleged advantages-A systematic review. J Oral Rehab 44(4): 261-290.

15. Lombardi RE (1977) Factors mediating against excellence in dental esthetics. J Prosthet Dent 38(3): 243-248.

16. Joda T, Brägger U, Gallucci G (2015) Systematic literature review of digital three-dimensional superimposition techniques to create virtual dental patients. Int J Oral Maxillofac Implants 30(2): 330-337. 
17. Zhang C, Liu T, Liao W, Yang T, Jiang L (2017) Computer-aided design of dental inlay restoration based on dual-factor constrained deformation. Advances in Engineering Software 114: 71-84.

18. Rengier F, Mehndiratta A, von Tengg-Kobligk H, Zechmann CM, Unterhinninghofen $\mathrm{R}$, et al. (2010) 3D printing based on imaging data: review of medical applications. Int J Comput Assist Radiol Surg 5(4) 335-341.

19. Burkhardt MA, Waser J, Milleret V, Gerber I, Emmert M, et al. (2016) Synergistic interactions of blood-borne immune cells, fibroblasts and extracellular matrix drive repair in an in vitro peri-implant wound healing model. Scientific reports 6: 21071.

20. Coachman C, Calamita MA, Coachman FG, Coachman RG, Sesma N (2017) Facially generated and cephalometric guided 3D digital design

ISSN: 2574-1241

DOI: $10.26717 / B J S T R .2018 .06 .001408$

Luca Ortensi. Biomed J Sci \& Tech Res

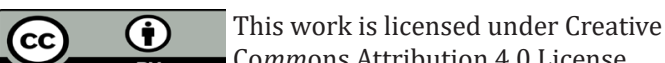

Submission Link: https://biomedres.us/submit-manuscript.php for complete mouth implant rehabilitation: A clinical report. J Prosthet Dent 117(5): 577-586.

21. Koutsoukis T, Zinelis S, Eliades G, Al-Wazzan K, Rifaiy MA, et al. (2015) Selective Laser Melting Technique of Co-Cr Dental Alloys: A Review of Structure and Properties and Comparative Analysis with Other Available Techniques. Journal of Prosthodontics 24(4): 303-312.

22. Goodacre BJ, Goodacre CJ, Baba NZ, Kattadiyil MT (2018) Comparison of denture tooth movement between CAD-CAM and conventional fabrication techniques. J Prosthet Dent 119(1): 108-115.

23. Montanari M, Bonato G, Ortensi L (2016) Oral Rehabilitation with Implant-Supported Overdenture and a New Protocol for Bar Passivation. Global Journal of Oral Science 2: 10-19.

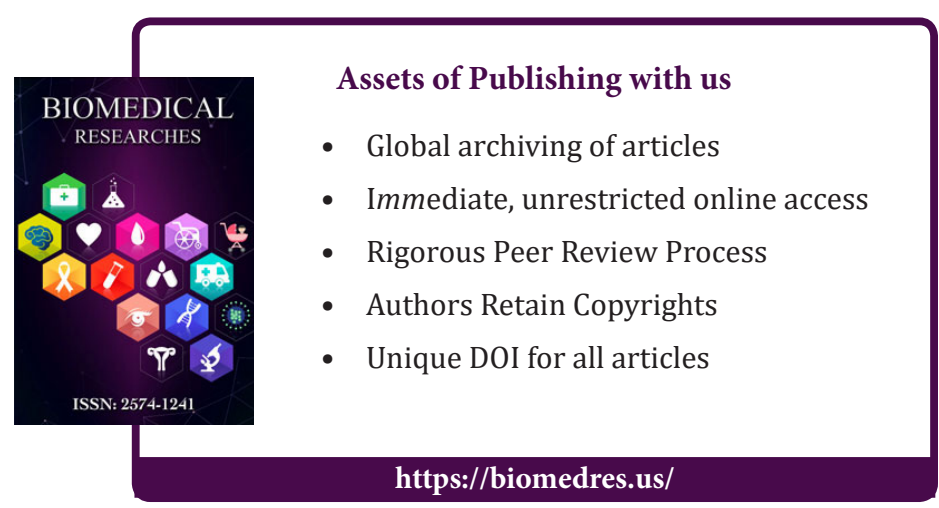

